\title{
BL Lacertae Objects: A Short Review
}

\author{
B. Kapanadze*1,2,3 \\ ${ }^{1}$ Ilia State University, Colokashvili Av. 3/5, Tbilisi, Georgia, 0162 \\ ${ }^{2}$ E. Kharadze National Astrophysical Observatory, Mt.Kanobili, Abastumani, Georgia, 0803 \\ ${ }^{3}$ INAF, Osservatorio Astronomico di Brera, Via E. Bianchi 46, 23807 Merate, Italy
}

\begin{abstract}
BL Lacertae objects (BLLs) constitute a class of active galactic nuclei (AGNs) with extreme observational features explained by non-thermal radiation from a relativistic jet nearly pointed along the observer's line-of-sight. Their spectral energy distribution (SED), extending over 17-19 orders of the frequency, is of non-thermal origin and shows a typical two-humped structure. The lower-energy component, ranging from the radio to X-rays, is explained via synchrotron radiation emitted by ultra-relativistic electrons/positrons/protons, to be initially accelerated via the Blandford-Znajek mechanism or magneto-hydrodynamic processes in the vicinity of a central supermassive black hole. Afterwards, the particles should undergo further acceleration to ultra-relativistic energies by means of different mechanisms (first and second-order Fermi processes, relativistic magnetic reconnection, shear acceleration, jet-star interaction etc.) locally, in the jet emission zone. Our intensive X-ray spectral study of TeV-detected, high-energy-peaked BLLs (HBLs) often show the signatures of an effective second-order Fermi (stochastic) acceleration close to the shock front, while the processes related to the first-order Fermi acceleration are relatively rarely presented. The TeV-undetected HBLs and low-energy-peaked BLLs (LBLs) mostly do not show the signatures of efficient stochastic acceleration in their jets. Concerning the higher-energy component, the most frequently considered scenario incorporates an inverse Compton (IC) scattering of synchrotron photons by their "parent" electron-positron population (synchrotron self-Compton model, SSC). However, this simple scenario sometimes is challenged by uncorrelated $\mathrm{X}$-ray and $\mathrm{TeV}$ variability, more easily explained by multizone SSC, external Compton (EC) and hadronic scenarios.
\end{abstract}

Keywords: (galaxies:) BL Lacertae objects: general

\section{INTRODUCTION}

Galaxies are generally divided into active and inactive, depending on the appearance of a central "engine". Active galactic Nuclei (AGNs), one of the most luminous sources in the universe, are galaxies with a strong and variable non-thermal emission, believed to be the result of accretion of mass onto a supermassive black hole lying in the center of the galaxy. They present unique observational signatures that cover the full electromagnetic spectrum over 15-19 of magnitude in frequency. The origin, internal structure and the nature of physical processes underlying the observed properties of AGN are among of the open problems in astrophysics.

AGNs are characterized by a rich phenomenology and, consequently, they are divided into several classes:

- Quasars

- Seyfert galaxies

- Radio galaxies

- Blazars

*bidzina kapanadze@iliauni.edu.ge 
Table 1. Major blazar monitoring programs ans samples

\begin{tabular}{|c|c|c|}
\hline $\begin{array}{c}\text { Program/Sample } \\
\text { (1) }\end{array}$ & $\begin{array}{c}\text { Frequencies/Bands } \\
(2)\end{array}$ & $\begin{array}{l}\text { Homepage } \\
(3)\end{array}$ \\
\hline Swift-XRT & X-ray $0.3-10 \mathrm{keV}$ & http://www.swift.psu.edu/monitoring/ \\
\hline Swift-BAT & $\mathrm{X}$-ray/ $\gamma$-ray $15-150 \mathrm{keV}$ & https://swift.gsfc.nasa.gov/results/transients/ \\
\hline Swift-UVOT & optical-UV & https://www.swift.ac.uk/analysis/uvot/ \\
\hline Fermi-LAT & $100 \mathrm{MeV}-300 \mathrm{GeV}$ & http://www.asdc.asi.it/fermi3fgl/ \\
\hline FACT & $\mathrm{TeV}$ & http://www.fact-project.org/ \\
\hline TUORLA & Optical $R$-band & http://users.utu.fi/kani/1m/index.html \\
\hline OVRO & $15 \mathrm{GHz}$ & http://www.astro.caltech.edu/ovroblazars/ \\
\hline UMRAO & $4.8,8,15 \mathrm{GHz}$ & https://dept.astro.lsa.umich.edu/datasets/umrao.php \\
\hline STEWARD & Optical & http://james.as.arizona.edu/ psmith/Fermi/ \\
\hline SMARTS & Optical & http://www.astro.yale.edu/smarts/glast/ \\
\hline MAXI & $\mathrm{X}$-ray $2-20 \mathrm{keV}$ & http://maxi.riken.jp/top/index.html \\
\hline RXTE-ASM & X-ray $1.5-12 \mathrm{keV}$ & http://xte.mit.edu/ASM_lc.html \\
\hline RXTE-PCA & $\mathrm{X}$-ray $2-60 \mathrm{keV}$ & https://heasarc.gsfc.nasa.gov/docs/xte/learning_center/pca.html \\
\hline PERKINS & Optical & https://www.bu.edu/blazars/VLBAproject.html \\
\hline GASP/WEBT & Optical & http://www. oato.inaf.it/blazars/webt/ \\
\hline CATALINA SKY SURVEY & Optical & http://nesssi.cacr.caltech.edu/catalina/Blazars/Blazar.html \\
\hline MOJAVE & $15 \mathrm{GHz}$ & http://www.physics.purdue.edu/MOJAVE/allsources.html \\
\hline KAIT & Optical & http://brando.astro.berkeley.edu/kait/agn/ \\
\hline ROBOPOL & Optical & http://robopol.org/ \\
\hline POLIMA & Optical & http://www.astrossp.unam.mx/blazars/ \\
\hline KANATA & Optical & http://adsabs.harvard.edu/abs/2011PASJ . . 63. . 639I \\
\hline TeVCAT & $\mathrm{TeV}$ & http://tevcat.uchicago.edu/ \\
\hline TANAMI & $8.4,22 \mathrm{GHz}$ & http://pulsar.sternwarte. uni-erlangen.de/tanami \\
\hline F-GAMMA & 2-200GHz, IR, optical & http://www.mpifr-bonn.mpg.de/div/vlbi/fgamma/fgamma.html \\
\hline METSAHOVI & $22,37 \mathrm{GHz}$ & http://www.metsahovi.fi/quasar/ \\
\hline MARMOT & $86 \mathrm{GHz}$, optical & http://www. astro.caltech.edu/marmot \\
\hline SMA Calibrator Database & $86,300,350 \mathrm{GHz}$ & http://sma1.sma.hawaii.edu/callist/callist.html \\
\hline ALMA Calibrator Database & $90,100,230,340 \mathrm{GHz}$ & https://almascience.eso.org/alma-data/calibrator-catalogue \\
\hline
\end{tabular}

- Quasi-stellar objects

Blazars form the most violently variable class of active galactic nuclei (AGNs), with timescales ranging from a few minutes (in the $\mathrm{keV}-\mathrm{TeV}$ energy range) to several years (radio to optical frequencies), variable radio-optical polarization, compact radio-structure and superluminal motion of some components and very broad continuum extending over the radio to the very high-energy (VHE, $E>100 \mathrm{GeV}) \gamma$-ray energy ranges. Moreover, the bolometric luminosity occasionally can reach a level of $10^{48} \mathrm{erg} \mathrm{s}^{-1}$ during the strong outbursts (see Falomo \& et al. (2014)). Blazars are divided into two sub-classes:

- BL Lacertae Objects (BLLs)

- Flat-spectrum radio quasars (FSRQs)

Additionally, BL Lacertae sources (BLLs) are characterized by featureless spectra, to be produced by a beamed non-thermal emission of the relativistic jet pointed to the observer. The prototype of these sources, BL Lacertae $\left(\alpha=22^{h} 02^{m} 43.29^{s}, \delta=+42^{\circ} 16^{\prime} 39.98^{\prime \prime}(2000)\right)$ was originally discovered and classified as an irregularly variable star by Hoffmeister (1929). After almost 40 years, its counterpart was detected by MacLeod \& Andrew (1968). There were the detections of similar objects with high optical polarization, high variability and featureless, power law-shaped optical spectral continuum next years. This led to the recognition of a separate group of the extragalactic objects hosted by elliptical galaxies and sharing some characteristics of quasars (Strittmatter \& et al. (1972)). The observational peculiarities of BLLs was later included in the coherent picture that foresees a relativistic jet viewed at a small angle as the main responsibility of all multi-wavelength BLL characteristics (Blandford \& Rees (1978) and references therein).

In this paper, we review some observations properties of BLLs provide the discussion about the underlying physical mechanisms. In Section 2, we list the basic major monitoring programs and catalogues, containing BLLs. The spectral energy distribution and emission mechanisms are described in Section 3. The statistics of BLL redshifts are provided in Section 4. The internal BLL structure and superluminal motions are treated in Section 5. Section 6 is devoted to the X-ray spectra and particle 


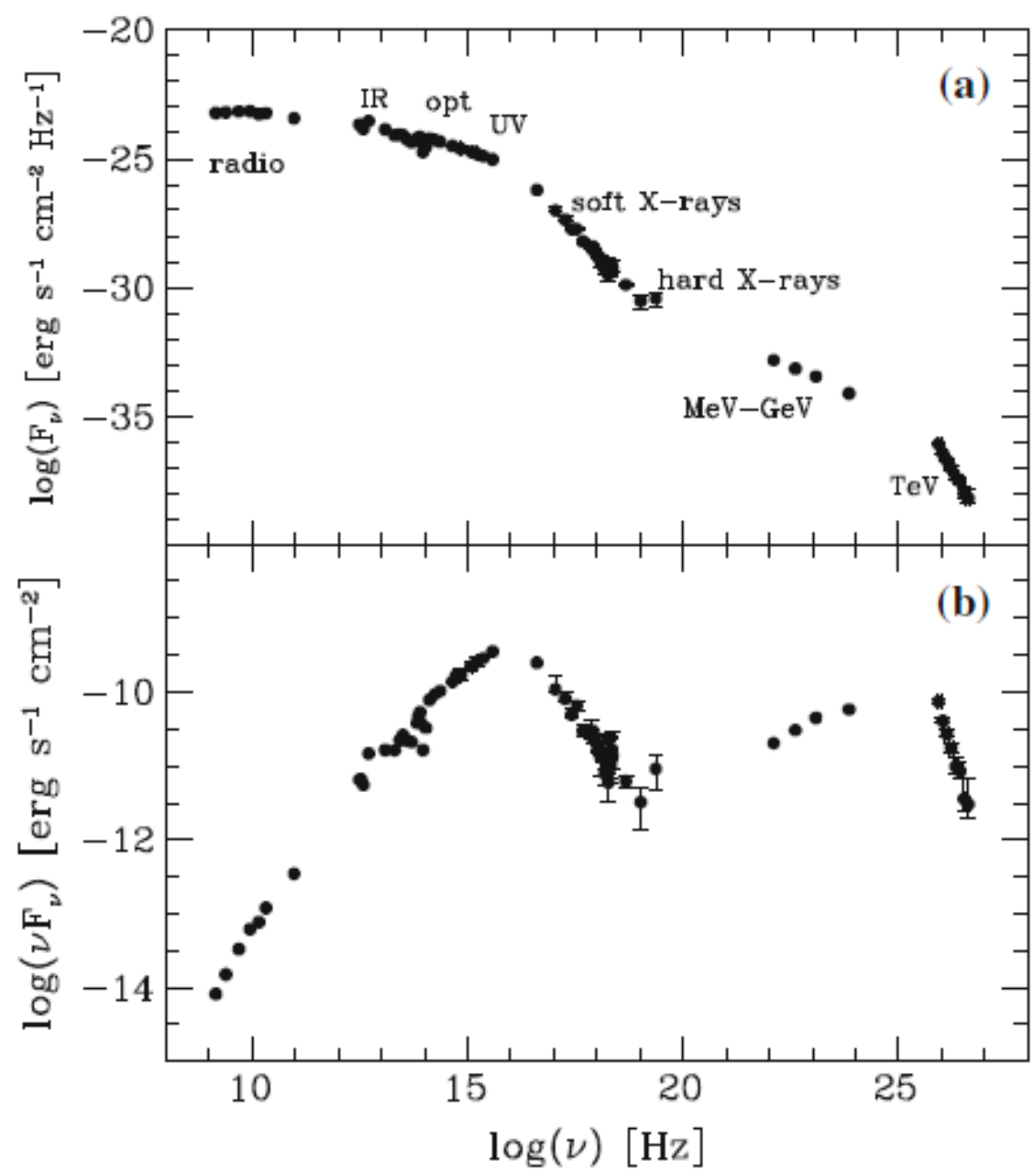

Figure 1. Broad-band spectrum of PKS 2155-304 ( $z=0.116)$ in the $\log \nu-F_{\nu}$ (panel(a)) and $\log \nu-$ $\log \nu F_{\nu}$ (panel (b)) representations (adapted from Treves et al. 2014). The latter emphasizes the frequencies at which most of the power is emitted.

acceleration processes in BLL jets. Finally, the flux variability of BLLs are discussed in Section 7, and give the summary of our review in Section 8.

\section{Catalogues and Major Monitoring Programmes}

There is a number of the Multifrequency (MWL) catalogues and surveys which contain BLLs. Namely,

- Roma BZCAT - Multi-frequency Catalogue of Blazars, 5th edition (Massaro \& et al. (2015))

- 4FGL - the fourth Fermi Large Area Telescope catalog of gamma-ray sources (Abdollahi \& et al (2019); previous versions: 1FHL, 2FHL, 3FHL, 1FGL, 2FGL, 3FGL)

- Veroncat - Veron Catalog of Quasars and AGN, 13th Edition; contains 133336 quasars, 1374 BLLs objects and 34231 other AGNs (Veron-Cetty \& Veron (2010))

- 1ES - Einstein Slew Survey (0.1-2 keV, 1992; previous versions: 1E, 2E; (Elvis et al. 1992): a catalog of 819 sources detected within the Einstein Slew Survey program of the X-ray sky. 
- Gaia - Catalogue of Gaia Sources (Evans et al. 2018, optical G-band, the second release)

- PBC - Palermo Swift-BAT hard X-ray catalogue obtained from the analysis of data acquired during the first 39 months of the Swift mission (15-150 keV energy range, Cusumano \& et al. (2010))

- 1RXS - ROSAT All-Sky Survey Bright Source Catalogue derived from the all-sky survey performed during the first half year (1990/91) of the ROSAT mission (Voges \& et al. (1999); previous versions: RBS, RX, 2RE)

- PKS - Parkes Survey of Southern Radio Sources, containing the $8400 \mathrm{MHz}$ flux densities for 1194 southern radio sources (Wright \& et al. (1991))

- $10 \mathrm{C}$ - the 10th Cambridge Survey (10C) od radio-sources at $15.7 \mathrm{GHz}$ using the Arcminute Microkelvin Imager Large Array, operated by the Cavendish Astrophysics Group at the University of Cambridge (Davies et al. 2011; previous versions: 1C-9C)

- SHBL - the multi-frequency sedentary survey is a flux-limited, statistically well-defined sample of highly X-ray dominated BLLs, which includes 150 sources (Giommi \& et al. (2005))

- 2XMM - Second XMM-Newton X-ray source catalogue (0.1-12 keV, 2009; https: / /xmmssc-www . star.le.ac.uk/Catalogue/2XMM/; previous version: XMMSL1)

- EGR - a catalog of point $\gamma$-ray sources detected by the EGRET detector on the Compton Gamma Ray Observatory (Casandjian \& Grenier (2008))

- PG - Palomar-Green Catalogue: a large area CCD survey for low surface brightness galaxies (Gregory \& et al. (1996))

- GB6 - 6th Green Bank Catalogue compiled by means of the Green Bank 4.85 GHz survey performed with the NRAO seven-beam receiver on the 91-m telescope during 1986 November and 1987 October (Gregory \& et al. (1996); previous versions: GB1-GB5, 87GB)

- $1 \mathrm{H}$ - the catalog of X-ray sources detected during the NRL Large Area Sky Survey (LASS) with the HEAO-1 satellite (Wood \& et al. (1984)).

- TXS - Texas survey of discrete radio sources between $-35.5 \mathrm{deg}$ and $71.5 \mathrm{deg}$ declination, which was carried out at $365 \mathrm{MHz}$ with the Texas Interferometer during 1974-1983 (Douglas \& et al. (1996)).

- JVAS - Jodrell-Bank VLA Astrometric Survey: a catalogue of 800 compact radio sources in the declination range 35-75 deg (5 GHz; Patnaik \& et al. (1992))

- S5 - the 5-GHz strong source survey between delineations $70^{\circ}$ and $90^{\circ}$ using the MPI 100$\mathrm{m}$ telescope, containing 476 sources with flux densities above 50 mJy (Kuehr \& et al. (1981); previous versions: $\mathrm{S} 4,1 \mathrm{Jy})$.

- OHIO - catalogues of radio sources (OJ, OM, etc. , depending on the source's position; 19641975; Rinsland \& et al. (1975))

- B2 - the second Bologna Survey, a list of 448 radio sources observed with the Bologna Northern Cross Telescope (408 MHz, 1970-1974; Fanti \& et al. (1974))

- QSO - Catalogue of Quasi-Stellar Objects (B-band, Hewitt \& Burbidge (1980))

The major BLL monitoring programs (in the radio, optical, X-ray etc. energy ranges) are listed in Table 1 along with the corresponding websites. Note that many of these programs are still ongoing, while the expired ones are characterized by rich, publicly available data archives. 


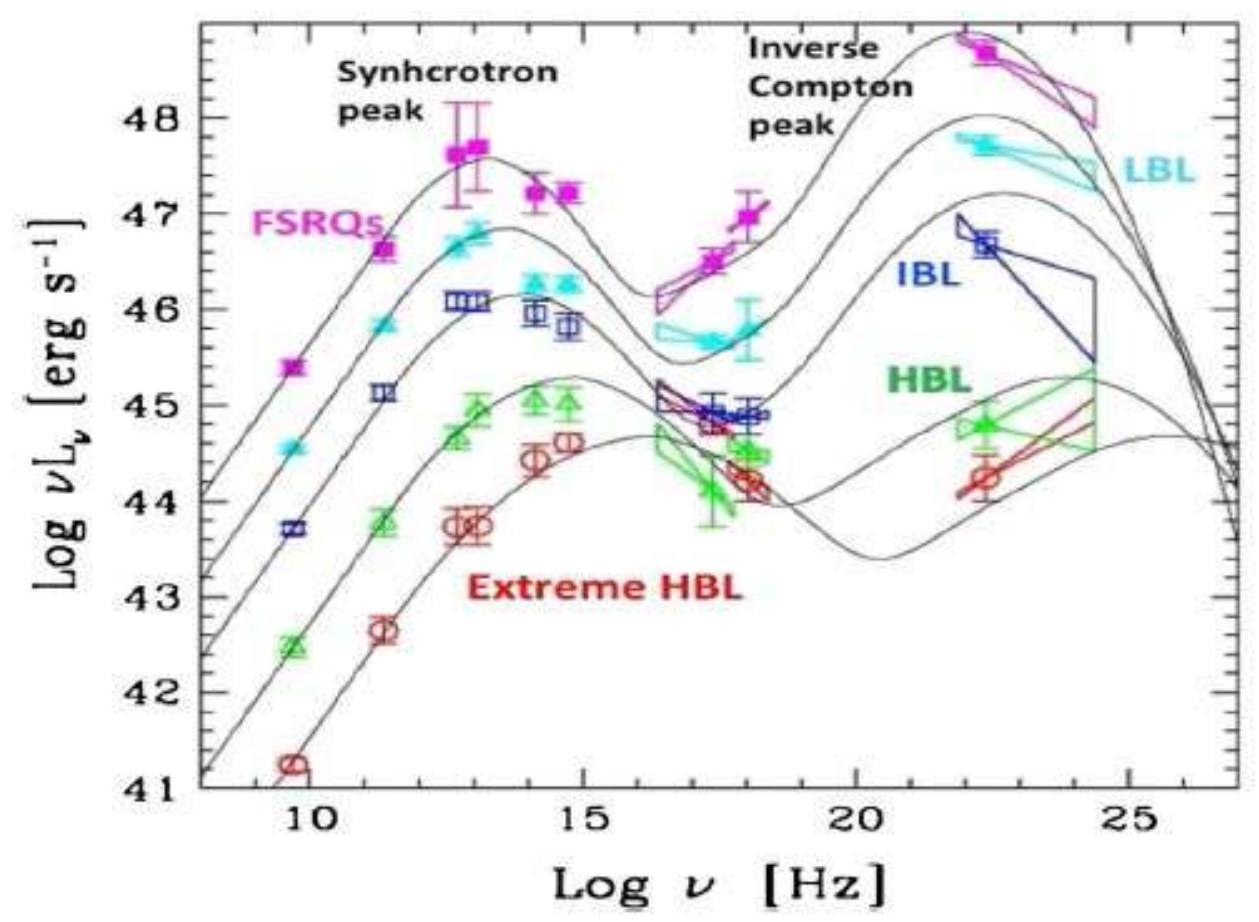

Figure 2. Broadband SEDs of the different BLL groups.

\section{Spectral Energy Distribution and Emission Mechanisms}

The optical spectra of BLLs are prominent for the absent or very weak spectral lines (belonging to host galaxy), and a similar situation is observed also in other spectral ranges. In the $\log \nu-\log \nu F_{\nu}$ plane, the broadband SED of BLLs shows two different components of a non-thermal origin (Figure 1):

- Lower-energy component extended from the radio to X-ray frequencies.

- Higher-energy component ranging from the X-ray up to very high-energy $\gamma$-rays.

Owing to the nonthermal, polarized nature of the lower-energy component, its origin is widely accepted as synchrotron emission of relativistic electrons (and, possibly, positrons and protons). On sub-parsec scales, jets should be pervaded by magnetic fields of the order of $0.1-10$ Gauss that may have tangled geometries and cause particles - leptons or hadrons - to radiate through the synchrotron mechanism (Treves et al. 2014).

However, the origin of the higher-energy SED component is still less clear and there are two different "competing" scenarios. Namely,

- In the leptonic scenario, this is ascribed to inverse Compton (IC) scattering of lower energy photons off the (ultra)relativistic electrons. Consequently, the gamma-ray spectral shape is determined by the high-energy tail of the electron distribution and by the shape of the spectrum of the scattered photons, which can be either optical-to-soft-X-ray synchrotron photons (synchrotron self-Compton, SSC) or photons external to the jet, like those from the accretion disk, broadline region and dust torus (external Compton, EC; see Böttcher (2007)).

- In hadronic models, both primary electrons and protons are accelerated to ultra-relativistic energies, with protons exceeding the threshold for photo-pion production on the soft photon field in the emission region. While the low-frequency emission is still due to synchrotron emission from primary electrons, the high-energy emission is dominated by proton synchrotron emission, neutral pion decay photons, synchrotron and Compton emission from secondary decay products of 
Table 2. The ranges of spectral parameters in HBLs during 1997-2016 (adapted from Kapanadze et al. 2018b).

\begin{tabular}{|c|c|c|c|c|c|}
\hline Source & $\mathrm{z}$ & $a$ & $\Gamma$ & $b$ & $E_{\mathrm{p}}$ \\
\hline Mrk 421 & 0.031 & $1.34(0.02)-3.02(0.06)$ & $1.68(0.02)-2.75(0.03)$ & $0.06(0.02)-0.64(0.06)$ & $0.002(0.001)-22.39(2.02)$ \\
\hline Mrk 501 & 0.034 & $1.39(0.06)-2.05(0.05)$ & $1.54(0.02)-2.22(0.04)$ & $0.12(0.01)-0.56(0.11)$ & $0.49(0.03)-101.6(23.7)$ \\
\hline 1ES $1959+650$ & 0.048 & $1.46(0.05)-2.37(0.03)$ & $1.71(0.02)-2.22(0.01)$ & $0.12(0.04)-0.98(0.12)$ & $0.12(0.04)-12.80(0.75)$ \\
\hline PKS 2155-304 & 0.116 & $2.04(0.02)-2.75(0.02)$ & $2.51(0.02)-2.81(0.02)$ & $0.11(0.02)-0.80(0.15)$ & $0.02(0.01)-0.89(0.10)$ \\
\hline PG $15553+113$ & $>0.4$ & $2.17(0.07)-2.21(0.03)$ & $2.09(0.03)-2.21(0.02)$ & $0.23(0.04)-0.63(0.08)$ & $0.51(0.11)-0.73(0.11)$ \\
\hline $1 \mathrm{ES} 1011+496$ & 0.212 & $1.72(0.04)-2.48(0.06)$ & $1.94(0.05)-2.50(0.07)$ & $0.10(0.04)-0.67(0.20)$ & $0.13(0.04)-5.01(0.68)$ \\
\hline H $1426+428$ & 0.129 & $1.68(0.02)-1.97(0.02)$ & $1.86(0.03)-2.22(0.11)$ & $0.12(0.02)-0.49(0.10)$ & $1.11(0.06)-21.95(0.60)$ \\
\hline H $1515+660$ & 0.701 & $1.27(0.10)-2.17(0.06)$ & $1.58(0.07)-2.53(0.18)$ & $0.12(0.08)-0.89(0.22)$ & $1.34(0.67)-3.53(1.52)$ \\
\hline PKS 2005-489 & 0.071 & $1.96(0.05)-3.12(0.02)$ & $2.00(0.03)-3.14(0.04)$ & $-0.23(0.06)-0.70(0.34)$ & $0.36(0.20)-1.25(0.22)$ \\
\hline PKS 0548-322 & 0.069 & $1.53(0.07)-1.87(0.05)$ & $1.49(0.03)-2.40(0.14)$ & $0.14(0.03)-0.52(011)$ & $1.77(0.21)-4.13(0.47)$ \\
\hline $1 \mathrm{ES} 0120+340$ & 0.272 & $1.39(0.20)-1.94(0.14)$ & $1.69(0.19)-1.94(0.14)$ & $0.33(0.22)-1.28(0.38)$ & $1.46(0.24)-3.31(0.44)$ \\
\hline 1ES 1218+304 & 0.184 & $1.89(0.05)-2.25(0.06)$ & $1.97(0.06)-2.25(0.06)$ & $0.39(0.11)-0.46(0.06)$ & $0.63(0.08)-1.32(0.16)$ \\
\hline 1ES $2344+514$ & 0.044 & $1.36(0.08)-1.87(0.11)$ & $1.72(0.01)-2.27(0.04)$ & $0.36(0.19)-0.83(0.33)$ & $1.35(0.36)-6.03(0.89)$ \\
\hline RBS 30 & 0.095 & $1.83(0.06)-1.89(0.05)$ & - & $0.36(0.19)-0.68(0.15)$ & $1.24(0.11)-1.44(0.29)$ \\
\hline $1 \mathrm{ES} 1421+582$ & 0.638 & $1.91(0.07)-1.98(0.10)$ & $1.77(0.10)-2.22($ & $0.37(0.17)-0.59(0.30)$ & $1.22(0.33)-1.42(0.30)$ \\
\hline 1ES $0033+595$ & 0.467 & $0.93(0.05)-1.60(0.16)$ & - & $0.36(0.07)-0.93(0.07)$ & $3.59(0.85)-6.47(0.95)$ \\
\hline 1ES $1727+502$ & 0.055 & $1.76(0.06)-2.12(0.08)$ & $1.90(0.05)-2.39(0.08)$ & $0.28(0.14)-0.65(0.18)$ & $0.81(0.14)-1.37(0.19)$ \\
\hline RGB J0710+591 & 0.125 & $1.62(0.06)-1.73(0.06)$ & $1.60(0.04)-1.85(0.02)$ & $0.17(0.11)-0.23(0.13)$ & $6.22(0.85)-6.70(0.93)$ \\
\hline 1ES 1101-232 & 0.186 & $1.64(0.08)-2.04(0.02)$ & $1.95(0.09)-1.99(0.10)$ & $0.17(0.03)-0.40(0.05)$ & $0.76(0.06)-3.50(0.33)$ \\
\hline $1 \mathrm{ES} 0229+200$ & 0.140 & $1.45(0.10)-1.60(0.10)$ & $1.43(0.06)-1.45(0.06)$ & $0.31(0.08)-0.44(0.21)$ & $4.22(0.47)-5.54(0.65)$ \\
\hline $1 \mathrm{ES} 0502+675$ & $>0.3$ & $1.63(0.11)-1.91(0.06)$ & - & $0.28(0.13)-0.70(0.20)$ & $1.28(0.18)-2.17(0.26)$ \\
\hline Mrk 180 & 0.045 & $1.60(0.06)-2.71(0.08)$ & $1.96(0.05)-2.59(0.07)$ & $0.17(012)-0.66(0.30)$ & $0.29(0.07)-8.11(0.85)$ \\
\hline RBS 1004 & 0.134 & $1.47(0.07)-1.74(0.07)$ & - & $0.38(0.19)-0.96(0.23)$ & $1.54(0.16)-4.26(1.57)$ \\
\hline RBS 76 & 0.610 & $2.11(0.05)-2.30(0.09)$ & $2.13(0.13)-2.29(0.09)$ & $0.49(0.21)-0.79(0.13)$ & $0.49(0.16)-0.79(0.13)$ \\
\hline BZBJ1137-1710 & 0.601 & $1.39(0.11)-1.67(0.13)$ & $1.48(0.15)-1.84(0.12)$ & $0.75(0.24)-1.09(0.39)$ & $1.42(0.58)-2.55(0.67)$ \\
\hline RBS 1457 & $>0.5$ & $1.47(0.12)-1.73(0.11)$ & $1.75(0.17)-2.19(0.16)$ & $0.74(0.28)-1.19(0.27)$ & $1.52(0.46)-1.67(0.48)$ \\
\hline 1ES $1533+535$ & $0.875 ?$ & $2.00(0.09)-2.22(0.06)$ & - & $0.34(0.18)-0.65(0.28)$ & $0.47(0.11)-1.00(0.29)$ \\
\hline BZBJ1341+3959 & 0.169 & $1.55(0.09)-1.64(0.07)$ & - & $0.66(0.21)-0.70(0.14)$ & $1.82(019)-2.20(0.38)$ \\
\hline RBS 1366 & 0.236 & $1.72(0.15)-2.03(0.11)$ & $1.90(0.09)$ & $0.39(0.34)-0.88(0.59)$ & $0.94(0.21)-2.08(1.24)$ \\
\hline $1 \mathrm{ES} 1440+122$ & 0.163 & $1.80(0.20)-1.83(0.11)$ & $2.15(0.18)$ & $0.32(0.16)-1.36(0.57)$ & $1.18(0.15)-1.93(0.52)$ \\
\hline B3 $2247+381$ & 0.119 & $1.80(0.09)-2.43(0.06)$ & $2.38(0.07)$ & $1.22(0.13)-0.29(0.18)$ & $0.19(0.02)-1.33(0.12)$ \\
\hline BZBJ0832+3300 & 0.672 & $1.47(0.13)-1.52(0.07)$ & $1.29(0.09)-1.81(0.09)$ & $0.37(0.15)-0.87(0.31)$ & $2.02(0.30)-4.45(0.52)$ \\
\hline
\end{tabular}

charged pions, and the output from pair cascades initiated by these high-energy emissions intrinsically absorbed by photon-photon pair production (see, e.g., Mannheim (1993) and references therein).

According to the position of the synchrotron SED peak position, BLLs are divided into four groups (see Figure 2):

- low-energy-peaked objects (LBLs) with the lower-energy peak at radio-IR frequencies

- Intermediate-energy-peaked objects (IBLs) peaking at optical frequencies

- High-energy-peaked objects (HBLs) with the synchrotron peak at UV-X-ray frequencies

- Ultra-High-energy-peaked objects (UHBLs) peaking at the frequencies corresponding to the energies $E>10 \mathrm{keV}$

Since the double-humped shape is present in all the aforementioned BLL groups, their relative intensities of the multifrequency emission differ significantly. According to Fossati \& et al. (2008), the SED shape define a continuum of properties, whereby (a) the most luminous sources peaking at lower frequencies; (ii) the peak frequency of the gamma-ray component correlates with the peak frequency of the lower energy one; (iii) the luminosity ratio between the higher- and lower-energy components increases with bolometric luminosity, which is evident in Figure 2. Within the so-called blazar sequence scenario, BLLs are the sources with lower luminosities and higher characteristic synchrotron and inverse Compton frequencies. The parameters, governing this sequence, are the cooling efficiency of the relativistic particles, the accretion efficiency and ultimately the mass of the central black hole, 
Table 3. The ranges of unabsorbed $0.3-2 \mathrm{keV}$ and $2-10 \mathrm{keV}$ fluxes (in units of $10^{-11} \mathrm{erg} \mathrm{cm}^{-2} \mathrm{~s}^{-1}$ ) of bright HBLs in different periods (adapted from Kapanadze et al. 2018).

\begin{tabular}{|c|c|c|c|c|c|}
\hline Per. & $F_{0.3-2 k e V}^{\min }$ & $F_{0.3-2 k e V}^{\max }$ & $F_{2-10 k e V}^{\min }$ & $F_{2-10 k e V}^{\max }$ & References \\
\hline \multicolumn{6}{|c|}{ Mrk 421} \\
\hline 1997 April - 2002 December & - & - & 4.10 & 93.20 & M04, R04 \\
\hline 2005 March - 2008 June & 15.17 & 261.82 & 3.50 & 312.35 & K18a \\
\hline 2009 January - 2012 December & 4.52 & 223.36 & 0.53 & 266.69 & TW \\
\hline 2013 January-May & 10.74 & 313.33 & 2.88 & 392.64 & K16a \\
\hline 2013 November - 2015 June & 19.72 & 166.72 & 4.37 & 140.93 & K17a \\
\hline \multicolumn{6}{|c|}{$1 \mathrm{ES} 1959+650$} \\
\hline 1997 May- 2002 November & - & - & 0.84 & 29.30 & G02, P05, M08 \\
\hline 2005 April - 2014 September & 4.22 & 30.50 & 1.91 & 24.3 & K16c \\
\hline 2015 August -2016 August & 11.17 & 34.67 & 6.17 & 50.58 & K16b \\
\hline \multicolumn{6}{|c|}{ Mrk 501} \\
\hline 1997 April 7 - 2007 April & - & - & 3.02 & 52.40 & M08 \\
\hline 2014 March-October & 7.21 & 33.19 & 6.04 & 53.20 & $\mathrm{~K} 17 \mathrm{~b}$ \\
\hline \multicolumn{6}{|c|}{ PKS 2155-304 } \\
\hline 1996 November - 2006 May & - & - & 1.22 & 8.23 & M08 \\
\hline 2005 November - 2012 October & 1.69 & 25.68 & 0.36 & 11.37 & K14 \\
\hline
\end{tabular}

while BLLs are accepted as the blazars having lower efficiencies and masses (Costamante \& et al. (2001); Treves et al. 2014).

Note that HBLs undergo a large change in the position of the synchrotron SED peak $E_{\mathrm{p}}$. For example, the nearby $\mathrm{TeV}$-detected and X-ray bright source Mrk 421 showed $E_{\mathrm{p}}<0.002 \mathrm{keV}$ in 2011 May (i.e. in the optical band; Kapanadze \& et al. (2018a)) and $E_{\mathrm{p}}=34_{-11}^{+22} \mathrm{keV}$ in 2006 June (Tramacere \& et al. (2009)), along with the tens of the spectra showing $E_{\mathrm{p}}>10 \mathrm{keV}$ (Kapanadze \& et al. (2016), Kapanadze \& et al. (2017a) Kapanadze \& et al. (2018a), Kapanadze \& et al. (2018b)). These cases show that Mrk 421 always was not an HBL source, but acts as an IBL or UHBL objects in low X-ray states and strong flares, respectively. A similar behaviour is observed also for many HBLs (see Table 2). The most extreme case was recorded for Mrk 501 during the dramatic X-ray flare in 1997 April when the position of the synchrotron SED peak moved from $0.94 \mathrm{keV}$ to beyond $100 \mathrm{keV}$ (i.e., to the $\gamma$-ray energies). While the presence of the synchrotron peak beyond $100 \mathrm{keV}$ was firmly established during that unprecedent event, it could not be constrained from above because of lack of the BeppoSAX sensitivity beyond $100 \mathrm{keV}$ (see Tavecchio \& et al. (2001)).

Due to the positions of the lower-energy and higher-energy peaks, BLLs are bright X-ray (see Table 3 and Figure 3 ) and $\gamma$-ray sources. Namely, they represent the most frequently detected class of the extragalactic $\mathrm{TeV}$ sources ( 65 out of 82 , see Table 4 ).

BLLs are one of the most frequently detected sources in the $100 \mathrm{MeV}-300 \mathrm{GeV}$ with Fermi-LAT and form one of the most important constituents of the 4FGL catalogue. Namely, 3131 out of 5090 4FGL sources are blasars including

- 1116 BLLs

- 686 FSRQs

- 1330 blazar candidates of uncertain type

The MWL campaigns on bright sources (e.g. Mrk 4211, Mrk 501) showed that X-ray and VHE emission are generally highly correlated down to sub-hour timescales with no evidence of significant lags (Aharonian \& et al. (2009), Fossati \& et al. (2008), Alecsic \& et al. (2015) etc.). This result provides a strong support to the one-zone SSC scenario, according to which both lower and higher energy BLL emissions are produced by a single electron population.

However, some MWL campaigns revealed the features which can not be explained within the one-zone SSC scenario. Namely, some BLLs showed 
Table 4. List of the TeV-detected BLLs (http://tevcat.uchicago.edu/).

\begin{tabular}{|c|c|c|c|c|c|c|c|}
\hline Source & $\mathrm{Z}$ & Type & $\begin{array}{c}\text { Detection } \\
\text { Year } \\
\end{array}$ & Source & $\mathrm{Z}$ & Type & $\begin{array}{c}\text { Detection } \\
\text { Year }\end{array}$ \\
\hline Mrk 421 & 0.031 & HBL & 1992 & H 2356-309 & 0.165 & HBL & 2006 \\
\hline Mrk 501 & 0.034 & $\mathrm{HBL}$ & 1996 & RX J0648.7+1516 & 0.179 & $\mathrm{HBL}$ & 2010 \\
\hline 1ES $2344+514$ & 0.044 & $\mathrm{HBL}$ & 1998 & 1ES $1218+304$ & 0.182 & $\mathrm{HBL}$ & 2006 \\
\hline Mrk 180 & 0.045 & $\mathrm{HBL}$ & 2006 & 1ES 1101-232 & 0.186 & HBL & 2006 \\
\hline 1ES $1959+650$ & 0.048 & $\mathrm{HBL}$ & 1999 & 1ES 0347-121 & 0.188 & $\mathrm{HBL}$ & 2007 \\
\hline AP Librae & 0.049 & LBL & 2010 & RBS 0413 & 0.190 & $\mathrm{HBL}$ & 2009 \\
\hline TXS $0210+515$ & 0.049 & HBL & 2019 & RBS 0723 & 0.198 & HBL & 2014 \\
\hline $1 \mathrm{ES} 2037+521$ & 0.053 & $\mathrm{HBL}$ & 2016 & 1ES $1011+496$ & 0.212 & $\mathrm{HBL}$ & 2007 \\
\hline 1ES $1727+502$ & 0.055 & $\mathrm{HBL}$ & 2011 & MS $1221.8+2452$ & 0.218 & $\mathrm{HBL}$ & 2013 \\
\hline PKS 1440-389 & 0.065 & HBL & 2012 & PKS 0301-243 & 0.266 & HBL & 2012 \\
\hline PGC 2402248 & 0.065 & $\mathrm{HBL}$ & 2018 & 1ES $0414+009$ & 0.287 & $\mathrm{HBL}$ & 2009 \\
\hline PKS 0548-322 & 0.065 & $\mathrm{HBL}$ & 2007 & OJ 287 & 0.306 & $\mathrm{HBL}$ & 2017 \\
\hline BL Lacertae & 0.069 & IBL & 2001 & 1RXS J023832.6-311658 & 0.322 & HBL & 2012 \\
\hline PKS 2005-489 & 0.071 & $\mathrm{HBL}$ & 2006 & OT 081 & 0.322 & LBL & 2016 \\
\hline RGB J0152+017 & 0.080 & $\mathrm{HBL}$ & 2008 & $3 \mathrm{C} 66 \mathrm{~A}$ & 0.34 & IBL & 1998 \\
\hline $1 \mathrm{ES} 1741+196$ & 0.084 & HBL & 2011 & PKS 0447-439 & 0.343 & HBL & 2009 \\
\hline SHBL J001355.9-185406 & 0.095 & $\mathrm{HBL}$ & 2010 & KUV 00311-1938 & 0.61 & $\mathrm{HBL}$ & 2012 \\
\hline W Comae & 0.102 & IBL & 2008 & 1ES $0033+595$ & - & $\mathrm{HBL}$ & 2011 \\
\hline 1ES 1312-423 & 0.105 & $\mathrm{HBL}$ & 2010 & $\mathrm{~S} 20109+22$ & - & IBL & 2015 \\
\hline PKS 2155-304 & 0.116 & HBL & 1999 & RGB J0136+391 & - & HBL & 2012 \\
\hline B3 $2247+381$ & 0.119 & HBL & 2010 & 1ES $0502+675$ & - & HBL & 2009 \\
\hline RGB J0710+591 & 0.125 & HBL & 2009 & VER J0521+211 & - & IBL & 2009 \\
\hline 1ES $0804+524$ & 0.128 & $\mathrm{HBL}$ & 2008 & $1 \mathrm{ES} 0647+250$ & - & HBL & 2011 \\
\hline H $1426+428$ & 0.129 & HBL & 2002 & S5 $0721+71$ & - & IBL & 2008 \\
\hline 1ES $1215+303$ & 0.131 & $\mathrm{HBL}$ & 2011 & PG $1553+113$ & - & $\mathrm{HBL}$ & 2006 \\
\hline RXJ1136.5+6737 & 0.134 & $\mathrm{HBL}$ & 2014 & H $1722+119$ & - & HBL & 2013 \\
\hline S3 $1227+25$ & 0.135 & IBL & 2015 & HESS J1943+213 & - & $\mathrm{HBL}$ & 2010 \\
\hline $1 \mathrm{ES} 0229+200$ & 0.140 & $\mathrm{HBL}$ & 2007 & MAGIC J2001+435 & - & IBL & 2010 \\
\hline 1RXS J101015.9-311909 & 0.143 & HBL & 2010 & RGB J2243+203 & - & $\mathrm{HBL}$ & 2014 \\
\hline 1ES $1440+122$ & 0.163 & $\mathrm{HBL}$ & 2010 & 1ES 2322-409 & - & $\mathrm{HBL}$ & 2018 \\
\hline
\end{tabular}
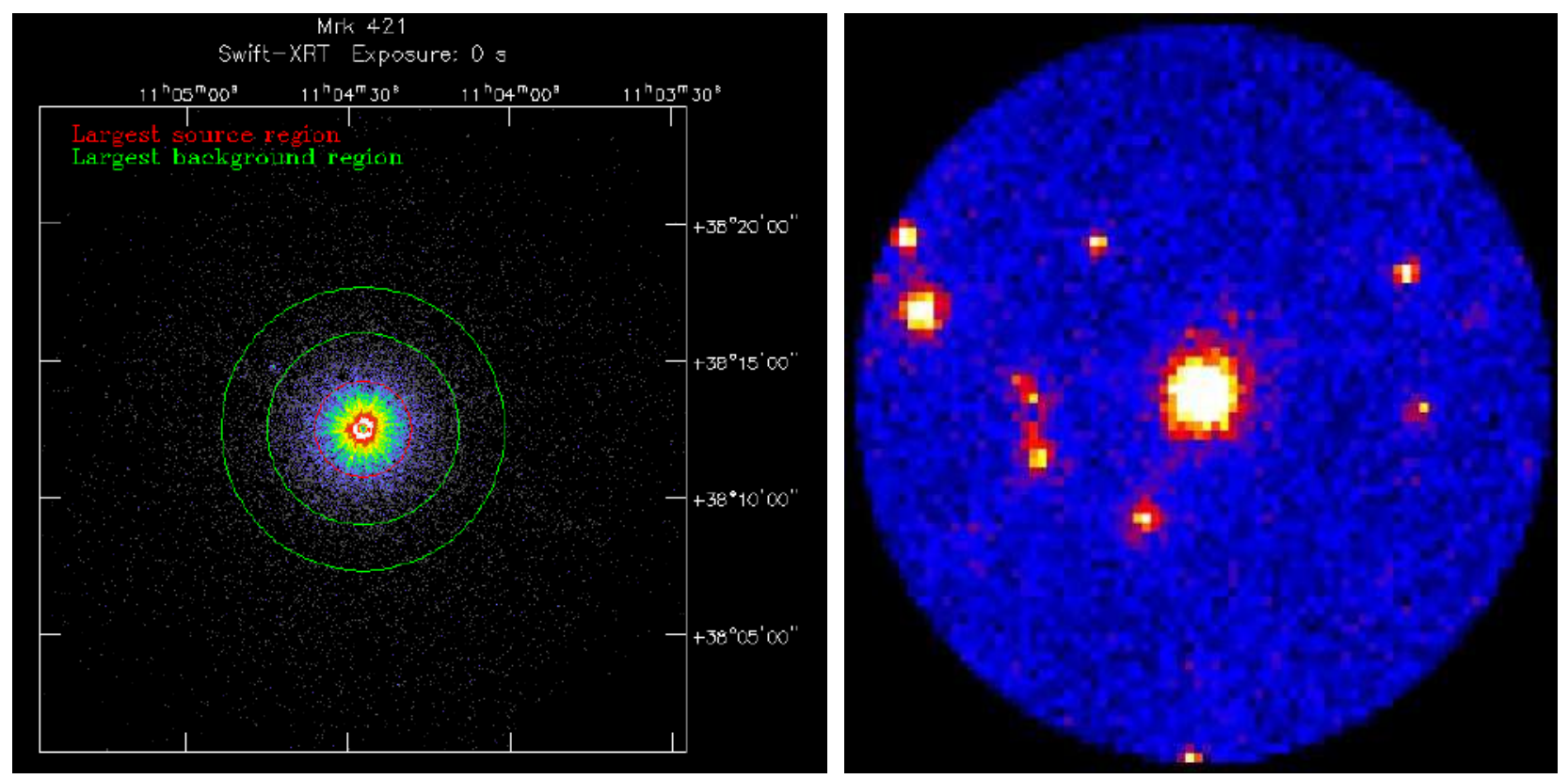

Figure 3. The $0.3-10 \mathrm{keV}$ (Swift-XRT; left panel) and $300 \mathrm{MeV}-300 \mathrm{GeV}$ (Fermi-LAT; right panel) images of Mrk 421. 
- VHE flares without any visible X-ray counterpart (e.g., an "orphan" flare of 1ES 1959+650 in 2002 June; Krawczynski \& et al. (2004)).

- X-Ray flares without VHE counterparts (e.g., in Mk 421; Blazejowski \& et al. (2005), Kapanadze \& et al. (2017a))

- off-sets between X-ray and TeV flares with more than a day (Blazejowski \& et al. (2005))

- More than a quadratic relation between the X-ray and VHE fluxes during some flares (in PKS 2155-304, Aharonian \& et al. (2009); in Mrk 501, Kapanadze \& et al. (2017b))

- Very fast (timescales of a few minutes) variability of some TeV blazars (see, e.g., Albert \& et al. (2007)) poses severe problems for single-zone models due to the required extremely high bulk Lorentz factors (Begelman et al. (2008))

There are a few observational evidences of the IC emission in BLLs. For example, more than a squared relation between the optical and Fermi-band variability in some epochs in BL Lacertae (Raiteri \& et al. (2013)). This feature can not explained within the one-zone SSC model, which predicting a quadratic relation between the synchrotron and IC emissions. Consequently, Raiteri \& et al. (2013) suggested a requirement of additional seed photons of non-jet origin (e.g., from the dusty torus). Moreover, some authors reported an an occasional detection of the $\gamma$-ray peak in the range between $\sim 5 \mathrm{GeV}$ and $\sim 100 \mathrm{GeV}$ in BL Lacertae, which was explained via the EC-upscatter of IR photons from dusty torus (Ghisellini \& Tavecchio (2015)).

Hadronic models are particularly important for EHBLs with exceptionally hard VHE emission (up to $\sim 10 \mathrm{TeV}$ ), which is very difficult to interpret the corresponding SED in the framework of the standard SSC model: extremely high Lorentz-factors of leptons and the reduction of the Klein-Nishina scattering cross-section necessarily entails soft spectra above $1 \mathrm{TeV}$ (Tavecchio (2014)). Morevover, some HBLs exhibit very hard X-ray and MeV-GeV spectra, e.g., Mrk 501 and 1ES 1959+650 showed the photon index values within the range of 1.1-1.7, which is easier to explain within the hadronic scenarios (Shukla \& et al. (2016), Kapanadze \& et al. (2017b), Kapanadze \& et al. (2018c)). Finally, an uncorrelated X-ray-VHE variability is "tolerated" by hadronic models (e.g., hadronic synchrotron mirror model, Böttcher (2005)).

On the other hand, there are some difficulties related to the use of hadronic models due to the reasons as follows:

- Requirement to accelerate protons to very high energies, together with the relatively low efficiency of the photo-meson and synchrotron proton emission generally implies higher magnetic fields and jet powers compared to leptonic models (Böttcher (2013)).

- It is very difficult to produce subhour flux variability within these models, since they require long cooling times (Aleksic et al. 2015).

\section{BLL Redshifts}

According to Roma-BZCAT, 1235 BL Lacs securely identified to date. The Redshifts are securely determined for 603 BLLs, ranging from from $z=0.020$ (NGC 2332; Marcha \& Caccianiga (2013)) to $z=1.283$ (PKS 2131-021; Drinkwater \& et al. (1997)). The distribution of the BLL redshift values and the observed ranges of the optical R-band magnitudes are provided in Figure 4.

103 BLLs have a tentative redshifts $z=0.07-1.57$ with $z>1$ for 11 sources and apparent optical R-band magnitudes of $R=14.7-21.0$ (see Table 3 for details). For the secure determination, we need to obtain deep optical spectra and identify absorption lines inherent to the host galaxy with higher

confidence levels. Moreover, the redshifts are still unknown for another 677 BLLs whose ranges of the $R$-band magnitude are provided in Table 3 . We see that these objects on average are fainter than the BLLs with the securely identified redshifts that prevents to obtain high-resolution optical spectra.

Among the BLLs with unknown redshifts, the most prominent is PG $1553+113$, which a bright source $(R \sim 14)$ and target of different multiwavelength campaigns, as well as a candidate object of 

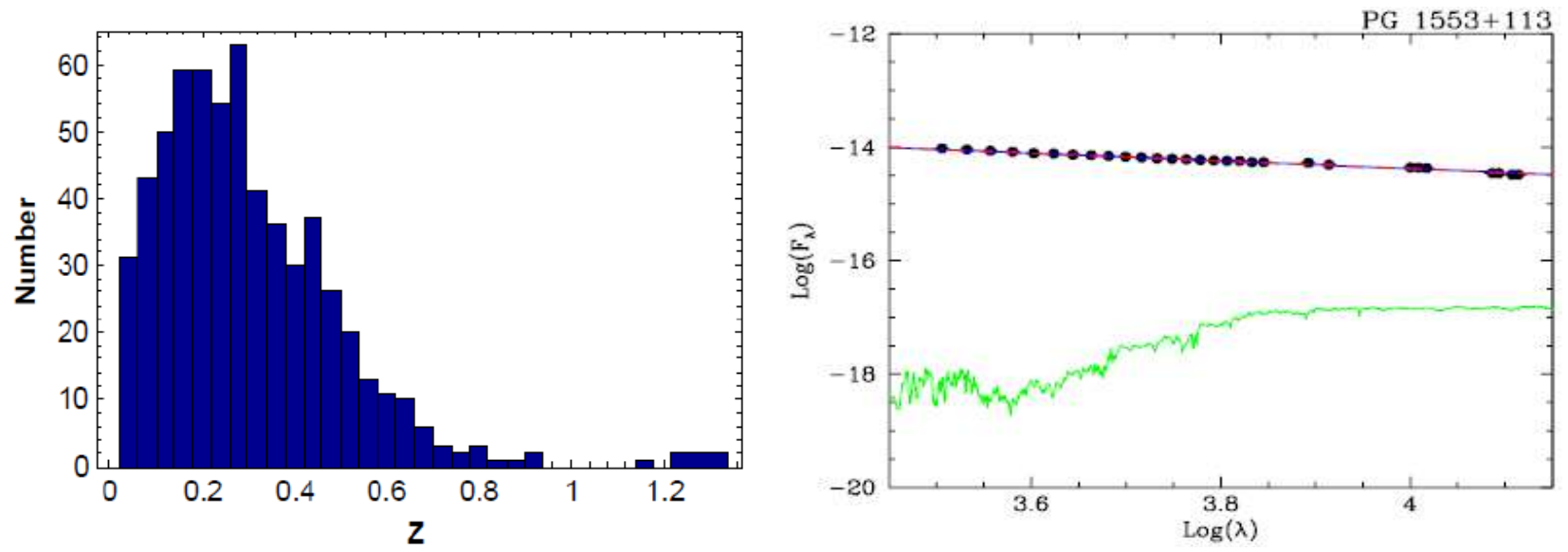

Figure 4. Redshift distribution of BLLs (left panel) and the UV-IR spectrum of PG 1553+113 (adapted from Landoni \& et al. (2015))

Table 5. Major blazar monitoring programs and samples.

\begin{tabular}{ccc|cc|cc}
\hline \multicolumn{2}{c}{ BLLs with Known Redshifts } & \multicolumn{2}{c}{ BLLs with Unknown Redshifts } & \multicolumn{2}{c}{ BLL Candidates } \\
\hline $\mathrm{z}$ & Number & $R$-mag & Number & $R$-mag & Number & $R$-mag \\
\hline$\leqslant 0.05$ & 25 & $8.0-16.5$ & 10 & $14.0-15.0$ & 0 & $14.0-15.0$ \\
$0.05-0.10$ & 50 & $9.8-17.5$ & 29 & $15.0-16.0$ & 1 & $15.0-16.0$ \\
$0.10-0.20$ & 138 & $12.8-18.9$ & 89 & $16.0-17.0$ & 5 & $16.0-17.0$ \\
$0.30-0.40$ & 92 & $15.0-21.6$ & 123 & $17.0-18.0$ & 8 & $17.0-18.0$ \\
$0.4-0.6$ & 119 & $15.5-20.8$ & 183 & $18.0-19.0$ & 14 & $18.0-19.0$ \\
$0.6-0.6$ & 25 & $16.5-20.8$ & 150 & $19.0-20.0$ & 20 & $19.0-20.0$ \\
$0.8-1.0$ & 7 & $15.0-20.0$ & 40 & $20.0-21.0$ & 3 & $20.0-21.0$ \\
$>1.0$ & 3 & $17.6-19.2$ & 4 & $21.0-23.0$ & 1 & $21.0-22.0$ \\
\hline
\end{tabular}

having a binary central BH. Originally, it appeared in the Palomar-Green catalogue in 1986 and was proved to have featureless spectra in the near UV and optical bands (see, e.g., Falomo et al. (1993)). The spectroscopic observations with the 8-m VLT telescope also confirmed a featureless nature of the spectrum and derived a lower limit for the source of $\mathrm{z}>0.1$ based on the lack of absorption features from the starlight component (Sbarufatti et al. (2006)). The X-Shooter spectroscopy at the ESO-VLT confirmed the featureless nature of the spectrum on the wide spectral range 3200-15000 Angstroms (see Figure 3) and the redshift lower limit $z \gtrsim 0.3$ was estimated (Landoni \& et al. (2015)).

Moreover, there are 88 BL Lac candidates which need further high-resolution spectral observations to exclude the presence of the emission lines with the equivalent width higher than 5 Angstroms (dividing line between BLLs and FSRQs; Falomo \& et al. (2014)). Among these sources, the redshifts determined for 26 objects $(z=0.112-0.598)$, while the tentative redshifts are provided for 10 sources $(z=0.085-1.738)$.

There are incorrect redshift values in Roma-BZCAT:

- $z=0.007$ for 5BZG J0204+4005, while the correct value is $z=0.072$ (Healey \& et al. (2008)).

- $z=1.34$ for 5BZB J0508+8432 (QSO B0454+844), although this is a lower limit to the redshift determined by Healey \& et al. (2008) and no further redshift measurement has been performed.

Moreover, several most nearby objects are included incorrectly among BLLs in ROMA-BZCAT. Namely,

- 5BZG J1719+4858 (MCG+08-31-04) situated at $z=0.024$ : there is no information about its BLL identification.

- 5BZG J1407-2701 (IC 4374) with $z=0.022$ which is a FSRQ source (Russel \& et al. (2013)).

- 5BZG J1148+5924 (NGC 3894) situated at $z=0.011$ : flat-spectrum weak-line radio galaxy (WLRG; Bondi \& et al. (2001)) 
Table 6. Comparison between the redshifts of BLLs and FSRQs.

\begin{tabular}{ccc}
\hline Parameter & BLLs & FSRQs \\
\hline$z_{\min }$ & 0.02 & 0.07 \\
$z_{\max }$ & 1.28 & 6.80 \\
$z_{\text {mean }}$ & 0.29 & 1.44 \\
$N_{\mathrm{z}>1}(\%)$ & $3(0.002)$ & $1269(67.5)$ \\
\hline
\end{tabular}

- 5BZG J0048+3157 ( NGC 262, MRK 348) with $z=0.015$ : in fact, this is a Seyfert 2 galaxy (Freitas \& et al. (2018))

- 5BZG J1945-5520 (NGC 6812, $z=0.015$ ): no information about the BLL identification is available.

- 5BZG J1840-7709 (ESO 45-11) with $z=0.018$ is a LINER galaxy (Kollatschny \& et al. (2008)).

- 5BZG J1336-0829 (NGC 5232) situated at $\mathrm{z}=0.023$ : no information about the BLL identification is available.

- 5BZGJ1407-2701 (PKS 1404-267, $z=0.022$ ) is a FSRQ source (Russel \& et al. (2013)).

If we compare the securely determined redshifts of BLLs and FSRQs, the latter turn out to be significantly distant objects (see Table 4). Namely,

- The maximum redshift of BLLs is $z=1.28$ (5BZB J2134-0153), while FSRQs are found at the distances as high as $z=6.80$ (5BZQ J1556+3517), representing one of the most distant sources in the universe detected to date.

- The mean redshift of FSRQs is about 5-times larger than that of BLLSs.

- A majority of FSRQs are detected at $z>1$, while only 3 out of 1235 BLLs are found at such distances.

However, the majority of the BLLs still do not have securely determined redshifts (as noted above), and the future deep spectral observations with large telescopes may change this statistics.

\section{Internal Structure and Superluminal Motions}

The innermost BLL region is still mostly unresolved by means of the direct observations, and we have to adopt its hypothetic structure as follows: there should be a central supermassive black hole (SMBH) of $10^{8}$ Sollar masses, surrounded by the accretion disc (AD) "feeding" the central "engine". Since the SMBH is spinning, it produces two opposite relativistic jets closely aligned to our line-ofsight (Blandford \& Rees (1978) and references therein; see Figure 5). No observational evidences for the presence of broad/narrow line region is found, in contrast to other AGN classes. The existence of a dust torus around SMBH is also still controversial.

The SMBH masses of BLLs are evaluated using (i) relationship between BH masses and host luminosity (Woo \& Urry (2002)); (ii) relationship between SMBH masses and stellar velocity dispersions (Falomo et al. 2003). The distribution of the SMBH masses obtained by means of the both methods are presented in Figure 6. As wee see, the SMBH masses of several BLLs are estimated to be higher than $10^{9} M_{\odot}$ on some occasion.

The mechanism of energy extraction and particle acceleration along the jet is not still completely understood. Two different scenarios of the BLL jet launching are generally considered:

- Blandford-Znajek mechanism (Blandford \& Znajek (1977)) which is based on the Penrose effect in accreting Kerr BH, magnetic field can be sustained by external currents. As such currents move along the horizon, the field lines are usually representing as originating from the event horizon and then being torqued by rotation. Consequently, there should be an outgoing electromagnetic flux of energy and momentum. 

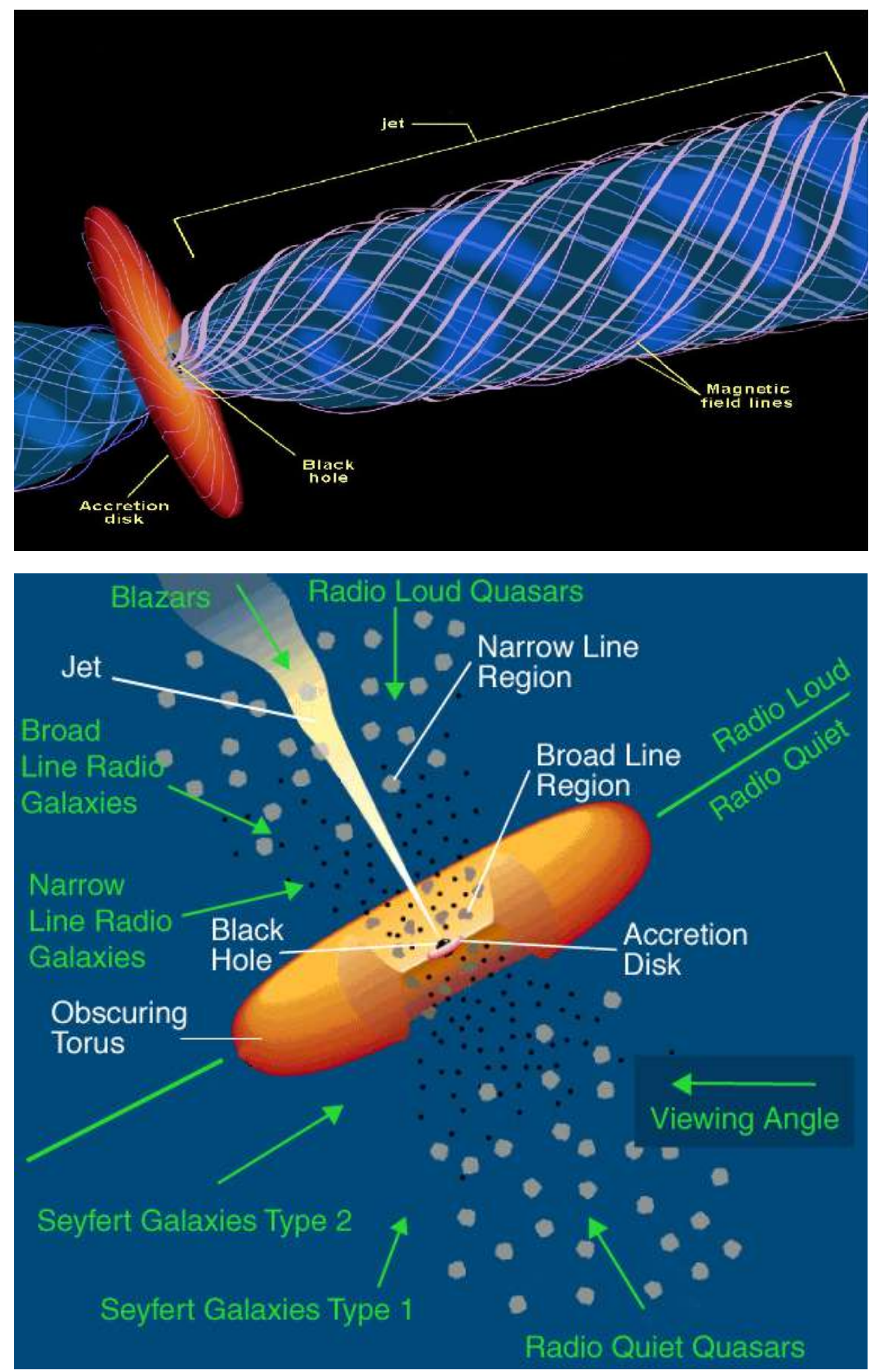

Figure 5. Hypothetic internal structure of a BLL source (www.universetoday.com/76443/ astronomy-without-a-telescope-blazar-jets/) 

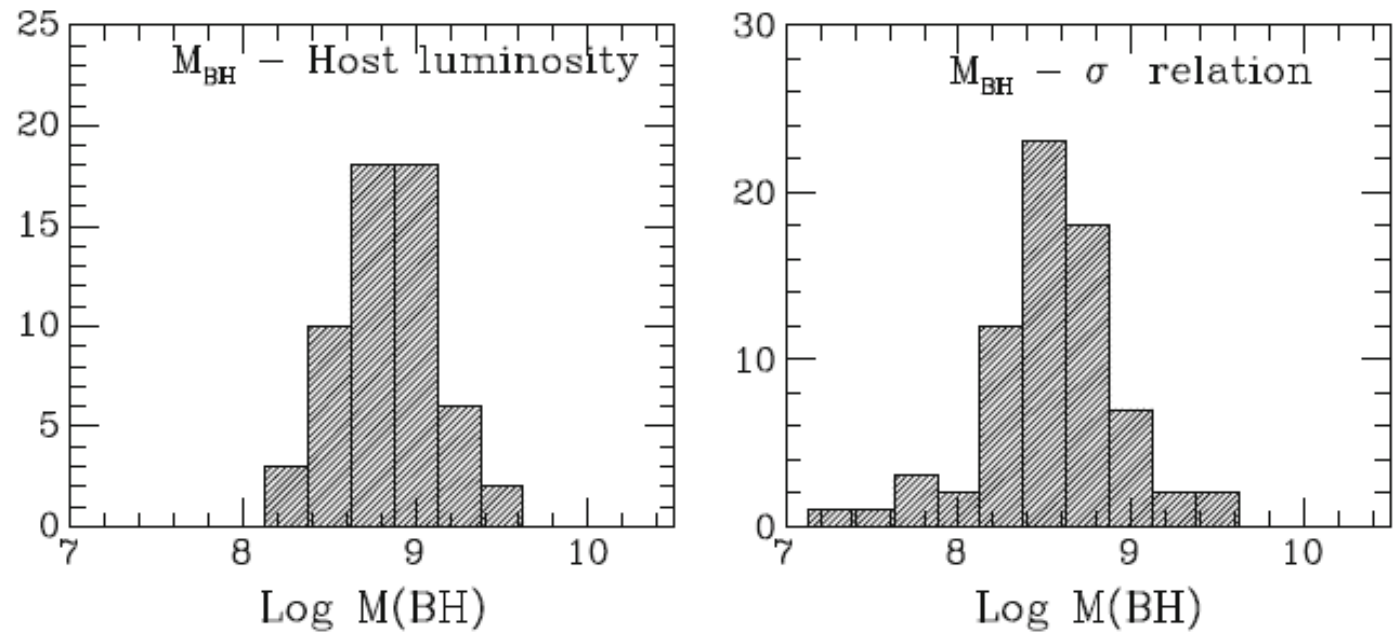

Figure 6. Distribution of BLL central BH $\beta$ masses derived from different relations (adapted from Treves et al. 2014).

- Blandford-Payne mechanism (Blandford \& Payne (1982)) - the BLL accretion disc should be threaded with magnetic field and torqued by its differential rotation. Consequently, it creates a magnetic coil that expels the accretion material and produces relativistic jets.

There is unanimous consensus on the fact that the extreme physical behavior of BLLs is related to the presence of a relativistic jet, which is closely aligned with the observer's direction - the estimated angle between the jet axis and observer's line-of-sight $\theta \lesssim 10$ deg (Begelman et al. (2008)). The highly relativistic kinematic regime and the small viewing angle produce a Doppler boosting of the observed emission as follows (Schneider (2006)):

$$
F_{\text {obs }}=\delta^{3+\alpha} F_{\text {int }} \text {, }
$$

where $F_{\text {obs }}$ is the intrinsic emission generated in the jet emission zone; the Doppler factor

$$
\delta=\frac{1}{\Gamma(1-\beta \cos \theta)},
$$

with Lorentz factor

$$
\Gamma=\frac{1}{\sqrt{\left(1-\beta^{2}\right)}}
$$

and $\beta=V / c ; V$ - the jet bulk speed; $c$ - speed of light in vacuum. In the BLL jets, the hot plasma moves with the velocities $\Gamma \sim 10$ and can become as high as as high as $\Gamma=50$ during strong X-ray and $\gamma$-ray flares (Begelman et al. (2008)). On the other hand, a small viewing angle and high jet bulk speed lead to the observation of some radio components separation from the radio-core with the apparent velocity

$$
V_{\text {app }}=\frac{V \sin \theta}{1-\beta \cos \theta},
$$

which can be superluminal for the given angle $\theta$, if

$$
\beta>\frac{1}{\sin \theta+\cos \theta} .
$$

Figure 7 presents an example of the superluminal motion of the IBL source $3 \mathrm{C} 66 \mathrm{~A}$ with $V_{\text {app }} \approx 30 c$ observed during 1992-1998, as well as the distribution of the superluminal $V_{\text {app }}$ values obtained for BLLs with Very Large Baseline Interferometry (VLBI) observations.

As noted above, the observed flux increases highly due to the Doppler boosting and allows us to detect BLLs at significantly larger distances. On the other hand, their detection probability declines 

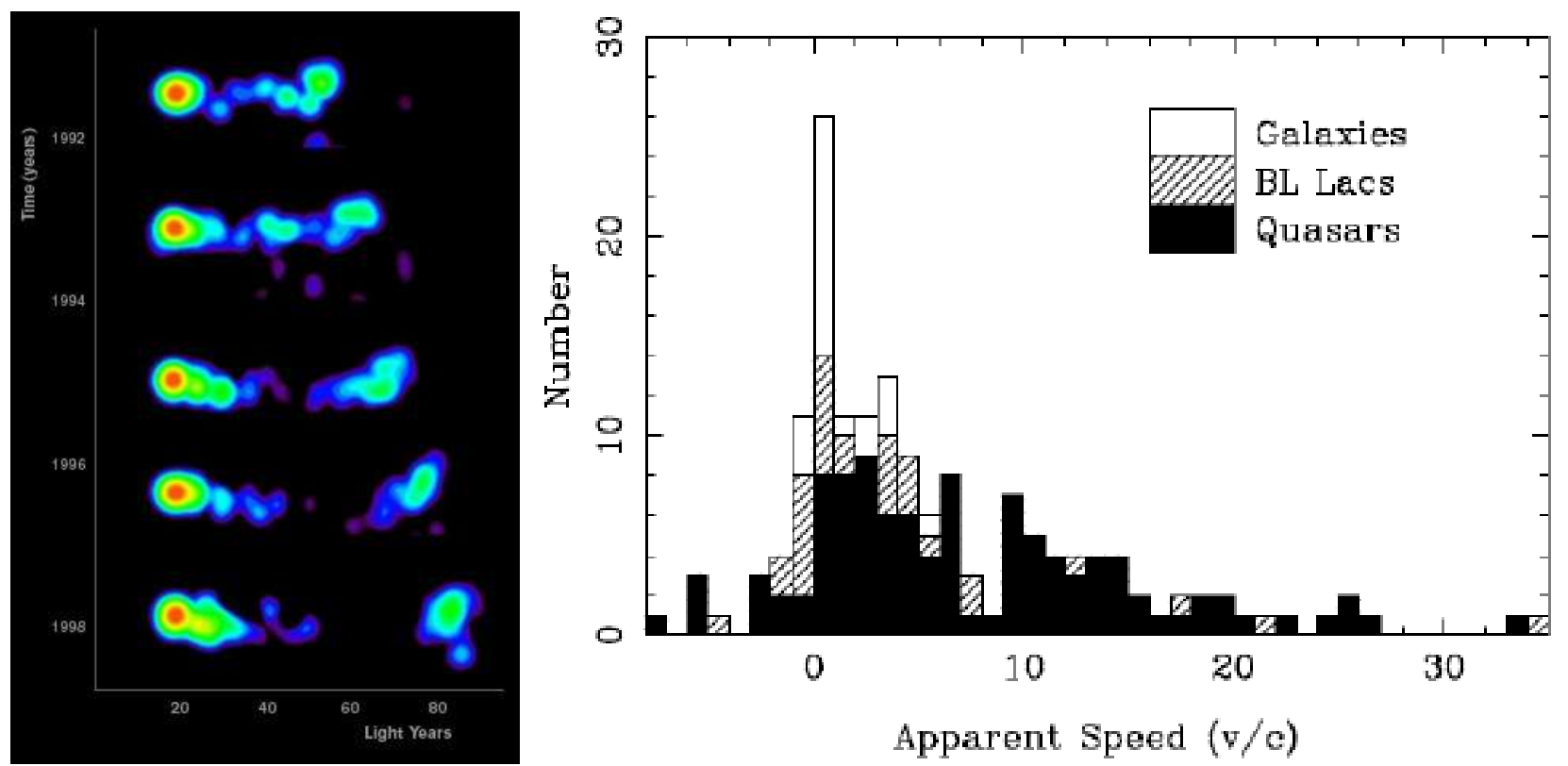

Figure 7. Superluminal motion of 3C 66A (left panel) and distribution of the superluminal motions observed for BLLs (right panel; adapted from Kellerman et al. 2003).

due to this effect - we can not reveal whether the particular elliptical galaxy possesses an BLL-type active nucleus if the angle between the jet axis and our line-of-sight is high enough. Consequently, it is impossible to evaluate an intrinsic number of the BLL sources in the universe and draw any firm conclusion whether an BLL-type AGN is one of the evolutionary stages of each elliptical galaxy.

\section{X-Ray Spectra and Particle acceleration Processes in BLL jets}

Generally, the X-ray spectra of BLLs are fitted with the theoretical models as follows:

- Simple power-law

$$
F(E)=K E^{-\Gamma},
$$

with $\Gamma$, the photon index throughout the entire spectral range

- broken power-law

$$
\begin{gathered}
F(E)=K E^{-\Gamma_{1}}, E \leq E_{\mathrm{br}} \\
F(E)=K E_{\mathrm{br}}^{\Gamma_{2}-\Gamma_{1}}(E / 1 k e V)^{-\Gamma_{2}}, E>E_{\mathrm{br}},
\end{gathered}
$$

with $E_{\mathrm{br}}$ : break point for the energy in $\mathrm{keV}, \Gamma_{1}$ : photon index for $E \leq E_{\mathrm{br}}, \Gamma_{2}$ : photon index for $E \leq E_{\mathrm{br}}$.

- log-parabolic model (Massaro \& et al. (2004))

$$
\left.F(E)=K\left(E / E_{1}\right)^{-(a+b l o g}\left(E / E_{1}\right)\right),
$$

with $E_{1}$, the reference energy; $a$, the photon index at the energy $E_{1} ; b$, the curvature parameter; $K$, the normalization factor. The position and the height of the synchrotron SED peak are calculated as

$$
\begin{gathered}
E_{\mathrm{p}}=10^{(2-a) / 2 b} \mathrm{keV} \\
S_{\mathrm{p}}=1.6 \times 10^{-9} K 10^{(2-a)^{2} / 4 b} \quad \mathrm{erg} \mathrm{cm}^{-2} \mathrm{~s}^{-1}
\end{gathered}
$$

Note that the X-ray spectra of the HBL and EHBL sources show a good fit with the log-parabolic model (see Figure 8 and Table 2 for the value ranges of different spectral parameters). Such spectra can be produced by the electron population with the logparabolic energy distribution. 

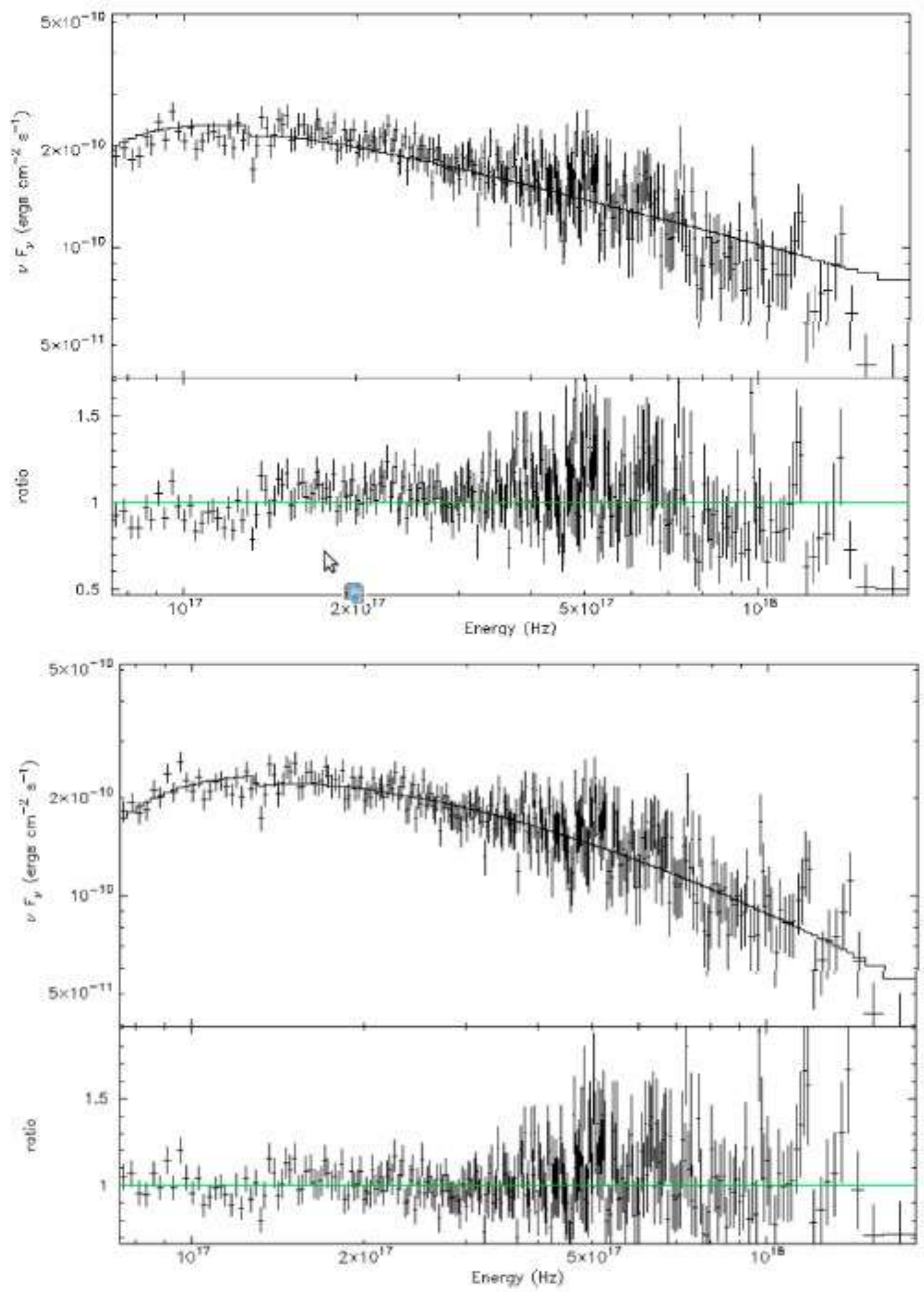

Figure 8. Simple power-law (upper panel) and log-parabolic (lower panel) fits of the spectrum derived from the Swift-XRT observation of Mrk 421 performed on 2009 March 24 (along with the distribution of the fit residuals with energy). We see that the fit with a simple power-law model produces a prominent trend in the residuals and is thus unsuitable for this spectrum. On the contrary, these residuals are distributed symmetrically around the value 1 in the case of the log-parabolic model, demonstrating a good fit of the model and the observed spectrum. 

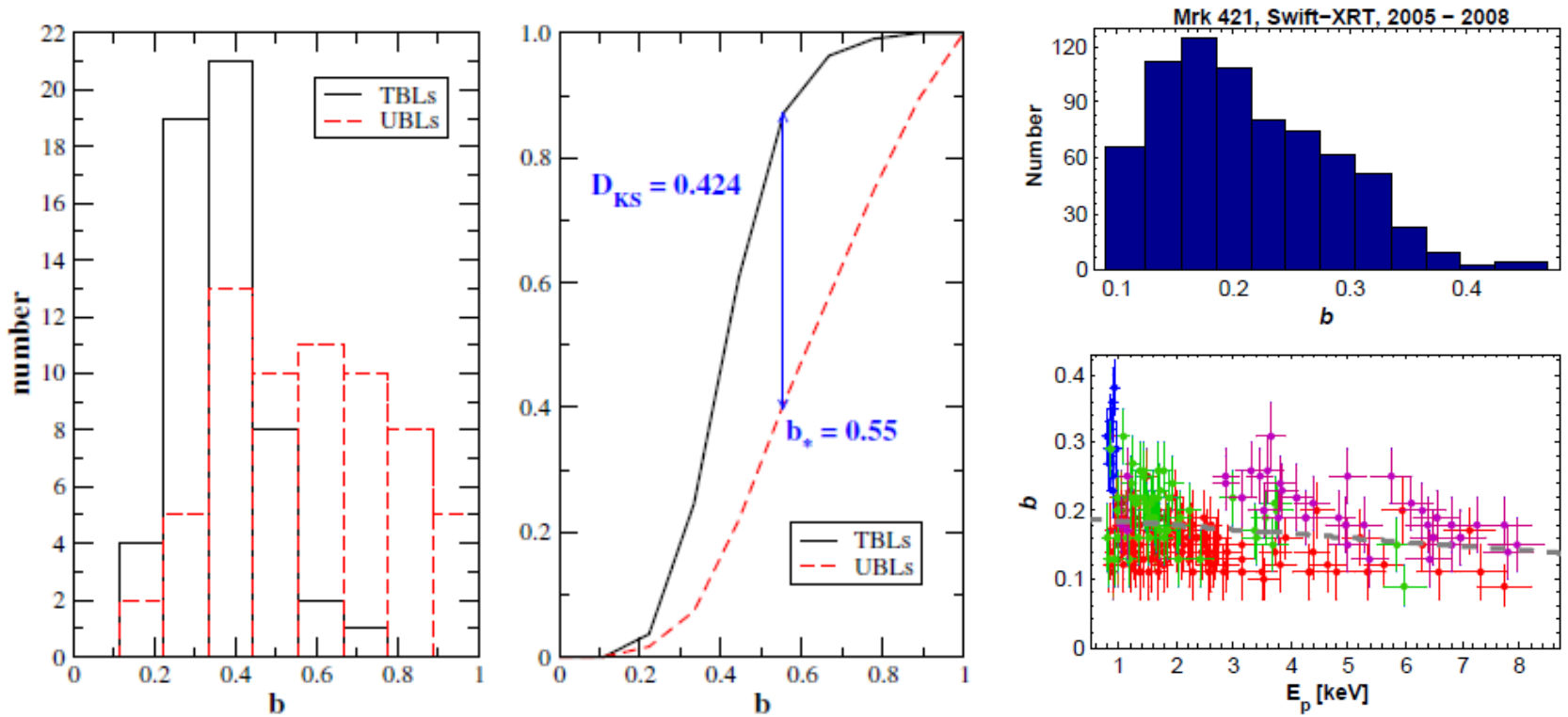

Figure 9. The distribution of the $b$ parameter values derived for TBLs sand UBLs: histogram (left panel) and cumulative normalized distribution (central panel; adapted from Massaro \& et al. (2011)). Distribution of these values and the $E_{\mathrm{p}}-b$ anti-correlation for Mrk 421 from the Swift-XRT observations performed during 2005-2008 (right panels; adapted from Kapanadze \& et al. (2018a)).

Generally, the energy spectrum of the BLL jet particles accelerated by some statistical mechanism, like that occurring in a shock wave, can be written as a power law (Massaro \& et al. (2004)):

$$
N(>\gamma)=N_{0}\left(\gamma / \gamma_{0}\right)^{-s+1},
$$

where $N(>\gamma)$ is the number of particles having a Lorentz factor higher than $\gamma ; s$ is the spectral index of the distribution defined by:

$$
s=-\frac{\log p}{\log \epsilon}+1,
$$

with $p$, the probability that a particle undergoes an acceleration step $i$ in which it has an energy gain equal to $\epsilon$. The latter parameter is generally assumed to be independent of the particle's energy:

$$
\gamma_{i}=\epsilon \gamma_{i-1}
$$

and

$$
N_{i}=p N_{i-1}=N_{0} p^{i} .
$$

A log-parabolic energy spectrum can be established when the condition $p$ is independent of energy is no more held and it can be described by a power-law relation as:

$$
p_{i}=g / \gamma_{i}^{q},
$$

where $g$ and $q$ are positive constants. In the case $q>0$, the probability for a particle to be accelerated is lower when its energy increases. Such a situation can occur in the jet region where the charged particles are confined by a magnetic field with a confinement efficiency decreasing for an rising gyration radius. Consequently, the probability of the particle's acceleration is lower when its energy increases, and the differential energy spectrum is given by

$$
N(\gamma) \sim \gamma / \gamma_{0}{ }^{-s-r \log \gamma / \gamma_{0}},
$$

with a linear relationship between the spectral index and curvature terms ( $s$ and $r$, respectively) as follows:

$$
s=-r(2 / q) \log g / \gamma_{0}-(q-2) / 2 .
$$




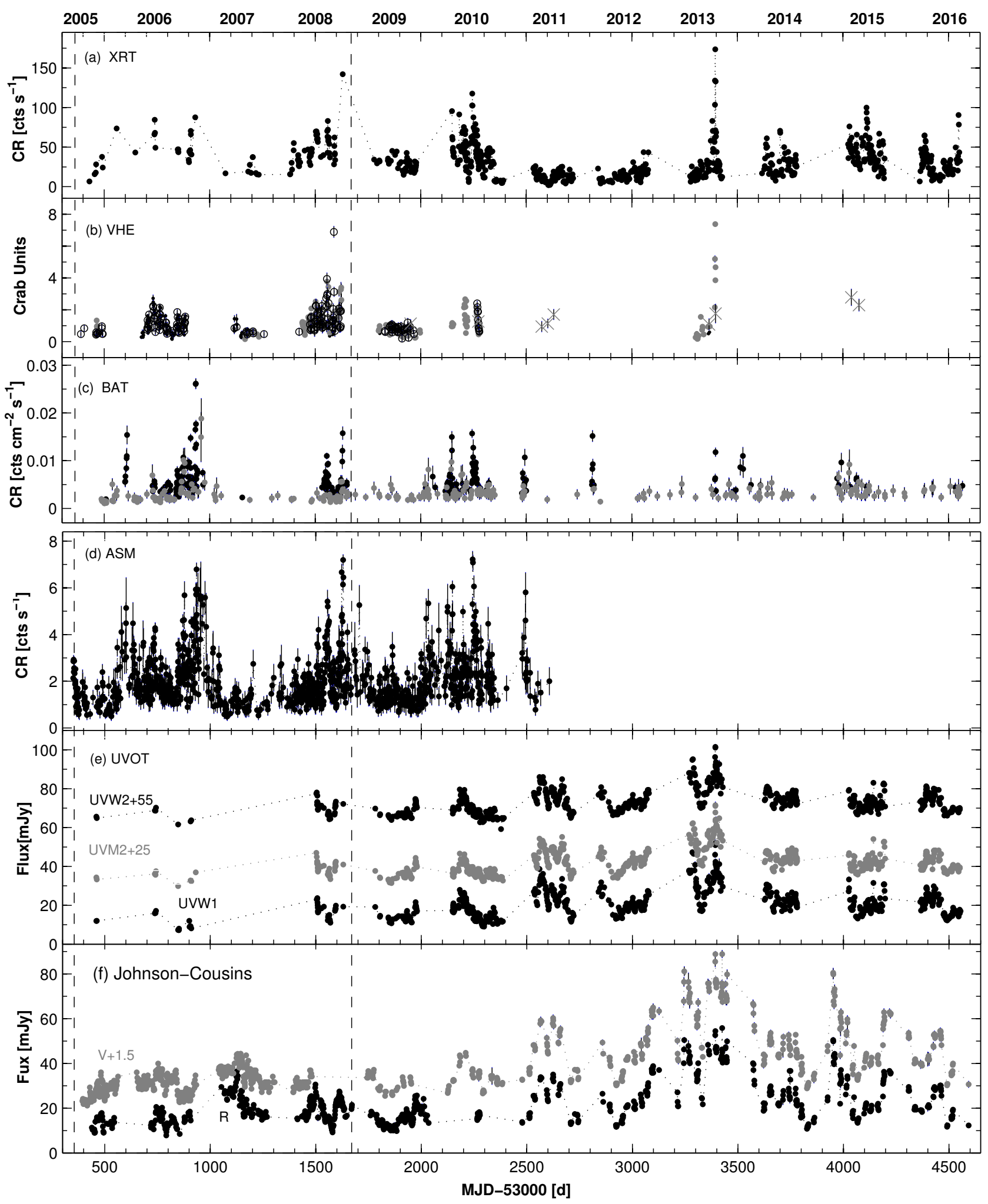

Figure 10. Historical light curves of Mrk 421 from the daily-binned MWL observations in 2005-2016 (adapted from Kapanadze \& et al. (2018a)).

Note that a larger volume or a higher magnetic field can make more efficient the particle confinement. In this case one can also expect that energetic particle can reach higher energies.

The synchrotron emission produced by this distribution is given by

$$
P_{S}(\nu) \propto\left(\nu / \nu_{0}\right)^{-\left(a+b \log \left(\nu / \nu_{0}\right)\right)},
$$

with $a=(s-1) / 2$ and $b=r / 4$, which can be derived using the aforementioned log-parabolic spectra 
model. Equation (16) demonstrates, that there should be a linear $a-b$ correlation in the case of the efficient first-order Fermi acceleration in the BLL jet. A positive is detected for nearby bright HBLs Mrk 421 and 1ES 1959+650 during the particular periods, using the densely-sampled observations performed with the X-Ray Telescope onboard the Swift satellite (Swift-XRT; Kapanadze \& et al. (2017a), Kapanadze \& et al. (2018a), Kapanadze \& et al. (2018b), Kapanadze \& et al. (2018c)). However, the detected $a-b$ correlations were generally weak in each interval that can be explained due to the presence of the sub-samples having different slopes in the $a-b$ plane, leading to a large scatter of the data points during the given period. Note that some sub-samples, corresponding to some X-ray flares, showed even a negative $a-b$ trend, which is expected when $g>\gamma_{0}$, i.e., there were electron populations with a very low initial energy $\gamma_{0}$ in the emission zone.

Furthermore, the co-existence of the second-order Fermi (stochastic) acceleration also could weaken the $a-b$ correlation, since this mechanism does not yield such a relation. The simulations of Katarzynski \& et al. (2006) demonstrated that electrons can be accelerated at the shock front via the first-order Fermi process and continue gaining energy via the stochastic mechanism into the shock downstream region. Afterwards, the accelerating particle will be able to re-cross the shock front and repeat the aforementioned acceleration cycle. Consequently, such combined energization process will not yield a strong $a-b$ correlation.

A shock acceleration is not the only statistical mechanism capable to produce a logparabolic energy distribution of charged particles. For example, the electron acceleration can occur in magnetohydrodynamical turbulence in which regions of magnetic field reconnection can develop in a very stochastic way (Massaro \& et al. (2006)). The stochastic (second-order Fermi) acceleration is related to the presence of a momentum diffusion term in the Fokker-Plank equation (Tramacere \& et al. (2011)). In this scenario, the diffusion term acts on the electron spectral shape, broadening the distribution via the inverse proportional relation between the curvature and diffusion terms:

$$
r \propto \frac{1}{D t} .
$$

In the case of the efficient stochastic acceleration, an anticorrelation between the peak energy $E_{\mathrm{p}}$ and the curvature parameter $b$ measured in the synchrotron SED is expected (Tramacere et al. 2009). However, the same correlation is expected also within the first-order Fermi mechanism, but the corresponding scatter plot is characterized by the slope which is different from that resulted in the case of the stochastic process. This circumstance explains a weak $E_{\mathrm{p}}-b$ anti-correlation, detected during the intense X-ray spectral study of nearby bright HBLs (see Kapanadze \& et al. (2016), Kapanadze \& et al. (2017a), Kapanadze \& et al. (2017b), Kapanadze \& et al. (2018a), Kapanadze \& et al. (2018b), Kapanadze \& et al. (2018c)); see Figure 9 for the corresponding example). The same studies also confirmed another expectation from the efficient stochastic acceleration: Massaro \& et al. (2011) showed that the electrons in the jets of TeV detected HBLs (so-called TBLs) should undergo a more efficient stochastic acceleration than in those of the TeV-undetected HBLs (UBLs; see Figure 9 ). They demonstrated that the synchrotron SEDs are relatively broad (i.e. the curvature is smaller with $b \sim 0.3)$ when the stochastic acceleration is more efficient while they are narrower $(b \sim 0.7)$ in the opposite case (for UHBLs). The corresponding example, demonstrating a low spectral curvature for Mrk 421 in the ime interval 2005-2008 is presented in Figure 9.

According to Tramacere \& et al. (2007), as the peak energy of the emission increases, the cooling timescale shortens and can compete with the acceleration timescales. Consequently, a positive $F_{0.3-10 \mathrm{keV}}-E_{\mathrm{p}}$ correlation, i.e., a trend of shifting the synchrotron SED peak to higher energies with rising X-ray flux is expected and confirmed in the aforementioned our X-ray spectral studies.

Relativistic magnetic reconnection represents another efficient convertor of magnetic energy into bulk motion and operate efficiently in the highly-magnetized jet areas (see, e.g., Sironi \& Spitkovsky (2014)). Although this mechanism provides a promising explanation for the long-wavelength (radioto-optical) flares, it importance for higher-energy flares is still unclear and further intense simulations required.

Shear acceleration is a Fermi mechanism without shock, wherever scattering centers flow at different speeds, even if the flows are parallel (e.g., longitudinal shear across the jet radius). Consequently, particles are intercepted by the difference between the fast core of the jet and the slower exterior 
(Rieger \& Duffy (2016)). Note that the shear acceleration can overcome radiative and non-radiative losses and work efficiently, when the pre-accelerated seed particles are available. Consequently, it can continue to accelerate the particles already energized by the first- or second-order Fermi mechanisms. However, a shear acceleration acts slowly and can not be important for very fast X-ray - TeV flares (Tammi \& Duffy (2009)).

\section{Flux Variability}

Flux variability of BLLs has been detected in most parts of the accessible electromagnetic spectrum, which is divided into different types according to their timescales (see, e.g., Gupta \& et al. (2016)):

- Long term variability (LTV), incorporating the brightness changes on monthly-yearly timescales (see Figure 10): usually explained by adopting the shock-in-jet scenario (Marscher \& Gear 1985 and references therein), which postulates that relativistic shocks in the jet cause particle acceleration and nonthermal emission, appearing as superluminal knots in VLBI maps. Relativistic shocks in the BLL jet can be triggered due to the intermittent variability in the physical properties in the innermost portion of the accretion disc. Such events may momentarily saturate the jet with extremely energetic plasma with much higher pressure than the steady jet plasma downstream. The high-energy plasma will sweep up slower moving material, forming a shock wave (Sokolov \& et al. (2004)).

- Short term variability (STV), lasting from a few days to a few weeks. Such event be triggered by the collision between a relativistic shock wave traveling down the jet and a more slowly moving or stationary compression, e.g., the core seen in VLBI images of BLLs (Spada \& et al. (2001)).

- Intraday variability (IDV) on timescales from several minutes to less than a day - can be produced by the shock interaction wit the smallest-scale turbulent jet inhomogeneities with strongest magnetic fields (Marscher (2014); Mizuno \& et al. (2014)), or by the instable processes in the SMBH vicinity and appearance of a "hot spot" on AD (Mangalam \& Wiita (1993)). The latter should be easier detectable in lower brightness states when the variable emission is not overwhelmed by the huge emission produced at the shock front during the long-term flare. On the contrary, the IDVs detected in higher states cab be explained more naturally within the shock-in-jet scenario.

Note that our detailed studies of Swift-XRT observations of nearby bright HBLs showed a dominance of the shock-in-jet scenario in triggering of 0.3-10 keV IDVS (Kapanadze \& et al. (2016), Kapanadze \& et al. (2017a), Kapanadze \& et al. (2017b), Kapanadze \& et al. (2018a), Kapanadze \& et al. (2018b), Kapanadze \& et al. (2018c)) shock-in-jet scenario. Note that our targets showed a series of the brightness halving and doubling events on timescales of a few hours. Such successive large brightness drop and rise events can be explained as a consequence of a shock passage through two inhomogenous areas with strong magnetic fields, which are separated by a region with significantly weaker field and lower particle density (yielding the generation of fewer X-ray photons). The most extreme behaviour was observed during the giant X-ray outburst of Mrk 421 in 2013 April, with several events showing $\tau_{\mathrm{d}}=1.2-7.2 \mathrm{hr}$ and $\tau_{\mathrm{h}}=1.0-3.5 \mathrm{hr}$ (Kapanadze \& et al. (2016)). The Variability character is generally erratic - BLLs change their amplitude, duration, maximum and minimum flux levels from flare to flare and can be characterized as a "red noise" - larger variability power at longer timescales.

The variability strength and timescales are highly dependent on subclass (LBL/IBL/HBL/UHBL): HBLs show the strongest and fastest variability in X-ray and TeV bands, while IBLs/LBLs are bright sources and undergo the strongest variability at the radio-optical and $\mathrm{MeV}-\mathrm{GeV}$ frequencies. Note that MWL variability of the majority of BLLs are still poorly investigated. 


\section{Summary}

- BLLs - an extreme classes of AGNs hosted by elliptical galaxies showing very wide range of distance: from $\mathrm{Z}=0.02$ to beyond $\mathrm{Z}=0.134$ with the distribution peak at $z \approx 0.28$. However, the intrinsic distribution peak may be quite different: there are $63 \%$ of BLLs with tentative or unknown redshifts, 88 BLL candidates and 227 blazars of uncertain type. Moreover, we can not evaluate the number of the undetected sources due to beaming effect or restricted instrumental capabilities. Consequently, it is impossible to draw any firm conclusion whether an BLL-type AGN is one of the evolutionary stages of each elliptical galaxy.

- The innermost structure of BLLs is still hypothetic since these regions are still unresolved via the direct observations. While the existence, properties and spatial scales are evaluated for the central SBBH, AD and two opposite jets, it is impossible to draw conclusion about the existence of BLR/NLR and dust torus, which are inherent to other AGN classes.

- BLLs represent one of the most extreme particle accelerators in the universe, showing an extreme range of broadband SED (up to 19 orders of frequencies, from radio to TeV) with two different non-thermal components. While the lower-energy one is firmly associated with the synchrotron emission of (ultra)relativistic charged particles gyrating in the jet magnetic field, there are different hypothetic mechanisms to be responsible for the appearance of the higher-energy SED component: one-zone SSC, multizone SSC, EC, or hadronic models. The intense MWL campaigns show that the model validity is variable from source to source and sometimes even one particular object requires different models from flare to flare.

- The most plausible for the initial particle acceleration scenarios invoke the Blandford-Znajek mechanism operating in the magnetospheres of the spinning Kerr SMBHs and the BlanfordPayne mechanism, related the magnetized accretion disc around SMBH. However, the $\mathrm{keV}-\mathrm{TeV}$ observations of BLLs demonstrate a requirement of the further particle acceleration within the jet. The latter is explained via the first-order Fermi process operating at the relativistic shock front moving downstream the jet. Moreover, the signatures of the stochastic (second-order) Fermi acceleration are also frequently observed for bright TeV-detected BLLs, to be related to the repeated particle scatter on the turbulent magnetic irregularities.

- Another jet-related acceleration mechanisms can be at work (with different contribution to diverse spectral bands and flares on various timescales): shear acceleration and relativistic magnetic reconnection. However, these possibilities are still relatively poorly investigated.

- BLLs are strongly variable objects on timescales from years (and, possibly, decades-long change in baseline emission) down to the events observed within a few minutes. The variability strength and timescales are highly dependent on subclass and the MWL variations of the BLL majority is still poorly investigated.

\section{References}

Abdollahi S., et al 2019, astro-ph/1902.10045v4

Aharonian et al. 2009, A\&A, 749, 502

Albert J., et al. 2007, ApJ, 862, 669

Alecsic J., et al. 2015, A\&A, 22, 578

Begelman M., Fabian A. C., Rees M. J., 2008, MNRAS, 384, L19

Blandford R. D., Payne D. G., 1982, MNRAS, 883, 199

Blandford R. D., Rees M. J., 1978, Pittsburgh Conf. on BL Lac Objects. Univ. Pittsburgh, Pittsburgh, PA, p. 328 
Blandford R. D., Znajek R. L., 1977, MNRAS, 433, 179

Blazejowski M., et al. 2005, ApJ, 130, 630

Bondi M., et al. 2001, MNRAS, 325, 1109

Böttcher M., 2005, ApJ, 176, 621

Böttcher M., 2007, ApSS, 95, 309

Böttcher M., 2013, ApJ, 54, 768

Casandjian J. M., Grenier I., 2008, A\&A, 849, 489

Costamante L., et al. 2001, A\&A, 512, 371

Cusumano G., et al. 2010, A\&A, 48, 510

Douglas J. N., et al. 1996, AJ, 1945, 111

Drinkwater M J., et al. 1997, MNRAS, 85, 284

Falomo R., et al. 2014, A\&AR, 22, 37

Falomo R., Bersanelli M., Bouchet P., Tanzi E. G., 1993, AJ, 11, 106

Fanti C., et al. 1974, AJ, 18, 147

Fossati G., et al. 2008, ApJ, 906, 677

Freitas I. C., et al. 2018, MNRAS, 2760, 476

Ghisellini G., Tavecchio F., 2015, MNRAS, 1060, 448

Giommi P., et al. 2005, A\&A, 385, 434

Gregory P. C., et al. 1996, ApJS, 427, 103

Gupta A. C., et al. 2016, MNRAS, 1127, 458

Healey S., et al. 2008, ApJS, 97, 175

Hewitt A., Burbidge G., 1980, ApJS, 57, 43

Hoffmeister C., 1929, AN, 233, 236

Kapanadze B., et al. 2016, ApJ, 102, 831

Kapanadze B., et al. 2017a, ApJ, 103, 848

Kapanadze B., et al. 2017b, MNRAS, 1655, 469

Kapanadze B., et al. 2018a, ApJ, 66, 854

Kapanadze B., et al. 2018b, ApJ, 68, 858

Kapanadze B., et al. 2018c, MNRAS, 407, 480

Katarzynski K., et al. 2006, A\&A, 47, 453

Kollatschny W., et al. 2008, A\&A, 897k, 484

Krawczynski H., et al. 2004, ApJ, 151, 601

Kuehr H., et al. 1981, AJ, 854, 86 
Landoni M., et al. 2015, A\&A, 126, 570

MacLeod J. M., Andrew B. H., 1968, ApL, 1, 243

Mangalam A. V., Wiita P. J., 1993, ApJ, 420, 406

Mannheim K., 1993, A\&A, 60, 269

Marcha M. J. M., Caccianiga A., 2013, MNRAS, 430, 2464

Marscher A. P., 2014, ApJ, 87, 780

Marscher A. P., Gear W. K., 1985, ApJ, 114, 298

Massaro E., et al. 2004, A\&A, 413, 489

Massaro E., et al. 2006, A\&A, 861, 448

Massaro E., et al. 2011, ApJ, L32, 742

Massaro E., et al. 2015, Ap\&SS, 75, 357

Mizuno Y., et al. 2014, MNRAS, 3490, 439

Patnaik A., et al. 1992, MNRAS, 655, 254

Raiteri C., et al. 2013, MNRAS, 1530, 436

Rieger F., Duffy P., 2016, ApJ, 34, 833

Rinsland C. P., et al. 1975, AJ, 759, 80

Russel H., et al. 2013, MNRAS, 530, 432

Sbarufatti B., Treves A., Falomo R., et al. 2006, AJ, 132, 1

Schneider P., 2006, in Extragalactic Astronomy and Cosmology: An Introduction, Springer, 188

Shukla A., et al. 2016, A\&A, 83, 591

Sironi L., Spitkovsky A., 2014, ApJ, L21, 783

Sokolov A., et al. 2004, ApJ, 725, 613

Spada M., et al. 2001, MNRAS, 1559, 325

Strittmatter P. A., et al. 1972, ApJ, 175L, 7

Tammi J., Duffy P., 2009, MNRAS, 1063, 393

Tavecchio F., 2014, MNRAS, 438, 3255

Tavecchio F., et al. 2001, ApJ, 725, 554

Tramacere A., et al. 2007, A\&A, 521, 466

Tramacere A., et al. 2009, A\&A, 879, 501

Tramacere A., et al. 2011, ApJ, 739, 66

Veron-Cetty M. P., Veron P., 2010, A\&A, 10, 518

Voges W., et al. 1999, A\&A, 389, 349

Woo J.-H., Urry C. M., 2002, ApJ, 530, 579

Wood K. S., et al. 1984, ApJS, 56, 507

Wright A. E., et al. 1991, MNRAS, 330, 251 